\title{
Insatisfação com a imagem corporal: avaliação comparativa da associação com estado nutricional em universitários
}

\author{
Body image dissatisfaction: comparative evaluation of the association with \\ nutritional status in university students
}

\author{
Raildo da Silva Coqueiro', Edio Luiz Petroski ${ }^{2}$, Andreia Pelegrini ${ }^{3}$, Aline Rodrigues Barbosa ${ }^{4}$ \\ ${ }^{1}$ Especialista. Estudante de Pós-Graduação, Universidade Federal de Santa Catarina (UFSC), Florianópolis, SC. Bolsista CNPq. ${ }^{2}$ Doutor. Professor \\ titular, Programa de Pós-Graduação em Educação Física, Núcleo de Pesquisa em Cineantropometria e Desempenho Humano (NuCIDH), UFSC. \\ ${ }^{3}$ Estudante de Pós-Graduação, UFSC. Bolsista CAPES. ${ }^{4}$ Doutora. Professora adjunta, Programa de Pós-Graduação em Educação Física, Núcleo de \\ Pesquisa em Cineantropometria e Desempenho Humano (NuCIDH), UFSC.
}

\begin{abstract}
Resumo
Introdução: O objetivo do estudo foi verificar a associação entre insatisfação com a imagem corporal e dois indicadores de estado nutricional em universitários brasileiros, assim como identificar o melhor preditor de insatisfação com a imagem corporal em universitários brasileiros. Métodos: Trata-se de um estudo transversal, realizado com 256 universitários (128 homens). Foram coletados dados demográficos (sexo e idade), antropométricos (massa corporal, estatura e espessura de dobras cutâneas) e de imagem corporal (silhueta atual e silhueta ideal). O índice de massa corporal $\left(<18,5 \mathrm{ou} \geq 25,0 \mathrm{~kg} / \mathrm{m}^{2}=\right.$ inadequado) e o somatório de espessura de cinco dobras cutâneas ( $\leq 30$ ou $\geq 62$ e $\leq 43$ ou $\geq 76 \mathrm{~mm}=$ inadequado, para homens e mulheres, respectivamente) foram derivados subseqüentemente. A análise incluiu estatística descritiva, teste de proporção, qui-quadrado e regressão logística.

Resultados: A média de idade dos indivíduos foi de 23,1 anos (desvio padrão = 5,3), e a freqüência de insatisfeitos com a imagem corporal foi de $78,8 \%$. A insatisfação com a imagem corporal não se associou ao sexo e ao índice de massa corporal. A análise multivariada demonstrou que a odds ratio associada ao somatório de espessura de cinco dobras cutâneas inadequado foi de 2,56 .

Conclusão: $O$ índice de massa corporal não foi determinante de insatisfação com a imagem corporal, enquanto o somatório de espessura de cinco dobras cutâneas mostrou-se um preditor significativo desse distúrbio, independentemente do sexo.
\end{abstract}

Descritores: Imagem corporal, estado nutricional, estudantes, auto-imagem.

\begin{abstract}
Introduction: The objective of this study was to investigate the association between body image dissatisfaction and two indicators of nutritional status in Brazilian university students, and also to identify the best predictor of body image dissatisfaction in Brazilian university students.

Methods: This was a cross-sectional study of 256 university students (128 males). Data were collected on demographics (age and sex), anthropometrics (body mass, stature and skinfold thickness), and body image (current silhouette and ideal silhouette). Body mass index (< 18.5 or $\geq 25.0 \mathrm{~kg} / \mathrm{m}^{2}=$ abnormal $)$ and the sum of five skinfolds $(\leq 30$ or $\geq 62$ and $\leq 43$ or $\geq 76 \mathrm{~mm}=$ abnormal, for men and women, respectively) were then calculated. The analysis included descriptive statistics, proportionality testing, chi-square test and logistic regression. Results: Mean age of the sample was 23.1 years (standard deviation $=5.3$ ) and the frequency of body image dissatisfaction was $78.8 \%$. Body image dissatisfaction was not associated with sex or body mass index. The multivariate analysis demonstrated that the odds ratio for abnormal sum of five skinfolds was 2.56.

Conclusion: Body mass index was not a determinant of body image dissatisfaction, whereas the sum of five skinfolds proved to be a significant predictor of this disorder, irrespective of sex.
\end{abstract}

Keywords: Body image, nutritional status, students, self-image.

Raildo da Silva Coqueiro, UFSC/CDS/NuCIDH, Campus Universitário, Trindade, Caixa Postal, 476, CEP 88040-900, Florianópolis, SC. Tel./Fax: (48) 3721.8562. E-mail: raildo@cds.ufsc.br 


\section{Introdução}

A percepção da imagem corporal pode ser definida como uma ilustração que se tem na mente acerca do tamanho, imagem e forma do corpo, e também dos sentimentos relacionados a essas características, bem como as partes que a constituem ${ }^{1}$. Dessa forma, pode ser entendida como um fenômeno de componentes afetivos, cognitivos, perceptivos e comportamentais ${ }^{2,3}$.

O componente subjetivo da imagem corporal se refere à insatisfação de uma pessoa com seu tamanho corporal e partes específicas do seu corpo. Assim, essa insatisfação envolve diversos fatores que se inter-relacionam, podendo ser citados: percepção da expectativa dos pais e amigos para ser mais magro ${ }^{4}$, localização geográfica da residência (grandes centros urbanos ou cidades menores) ${ }^{5}$, depressão e comportamentos de saúde (auto-estima, comportamento alimentar, uso de esteróides e dependência de exercício) ${ }^{3,6}$, percepção equivocada do peso corporal ${ }^{7}$, idade $^{6}$, razões pessoais para querer perder peso (aparência, saúde ou ${\text { estética })^{8} \text { e sexo }}^{9,10}$.

Enquanto alguns estudos sugerem que pessoas com excesso de peso apresentam maior insatisfação com a imagem corporal ${ }^{10,11}$, outros demonstram que mesmo naquelas com massa corporal adequada, a prevalência de insatisfação é bastante alta ${ }^{4}$ ou seja, a relação entre estado nutricional e insatisfação com a imagem corporal permanece não resolvida. As pesquisas que objetivaram verificar essa relação utilizaram apenas o índice de massa corporal (IMC) como medida de estado nutricional ${ }^{4,6,10-12}$.

A explicação mais utilizada para essas divergências em relação ao estado nutricional é que parece existir uma preocupação exagerada com a estética corporal, sendo que os padrões de beleza geralmente não correspondem aos padrões tidos como adequados para a saúde. Entretanto, pode-se atribuir como outra possível causa o fato do IMC não ser um indicador preciso da adiposidade corpora ${ }^{13,14}$. Nesse sentido, torna-se necessário verificar a associação entre a insatisfação com a imagem corporal e outras medidas de estado nutricional, a exemplo do somatório de espessuras de dobras cutâneas, que, além de manter a praticidade e baixo custo, reflete com melhor exatidão a adiposidade corporal dos indivíduos ${ }^{15}$.

Assim, os objetivos do presente estudo foram: a) verificar a associação entre insatisfação com a imagem corporal e dois indicadores de estado nutricional; b) identificar o melhor preditor de insatisfação com a imagem corporal em universitários brasileiros.

\section{Métodos}

Trata-se de um estudo transversal, realizado com universitários matriculados na disciplina Educação
Física Curricular (oferecida como disciplina optativa para os alunos de todos os cursos) de uma universidade pública da região de Florianópolis, estado de Santa Catarina, Brasil. Primeiramente, todos os professores foram convidados a colocar as suas turmas à disposição da pesquisa e liberar os seus respectivos horários para a coleta dos dados. Em seguida, os alunos das turmas em que os professores concordaram em participar do estudo foram informados com antecedência sobre os propósitos do estudo e datas da coleta dos dados. Dentro de cada turma participante, todos os alunos interessados foram incluídos na amostra e submetidos à avaliação.

Conforme informações da secretaria acadêmica, 1.034 alunos estavam regularmente matriculados na disciplina. Aceitaram participar do estudo 263 (25,4\%) universitários, sendo 133 do sexo masculino e 130 do sexo feminino. Após serem excluídos os indivíduos que não prestaram informações sobre a imagem corporal (cinco homens e duas mulheres), foram analisados dados de 256 participantes, distribuídos em proporções iguais entre os sexos. Todos os participantes foram convidados a preencher um questionário que incluía informações específicas sobre sexo, idade e percepção da imagem corporal.

O presente estudo foi aprovado pelo Comitê de Ética em Pesquisa da Universidade Federal de Santa Catarina. Os dados foram coletados após consentimento informado dos sujeitos, sendo-lhes assegurado o sigilo sobre as informações individuais.

As informações relacionadas à imagem corporal percebida foram obtidas utilizando-se a escala de silhueta proposta por Stunkard et al. ${ }^{16}$. Para a coleta dos dados, o conjunto de silhuetas era mostrado aos indivíduos e, então, realizadas as seguintes perguntas: Qual a silhueta que melhor representa a sua aparência física atualmente? Qual a silhueta que você gostaria de ter? O avaliador isentou-se de opinião na escolha das silhuetas. A verificação da insatisfação com a imagem corporal foi feita utilizando-se a discordância entre a silhueta atual e a silhueta ideal, classificando os indivíduos em satisfeitos e insatisfeitos.

Os dados antropométricos (massa corporal, estatura e espessura de dobras cutâneas) foram mensurados por dois avaliadores experientes, segundo procedimentos padronizados ${ }^{17,18}$. A medida da massa corporal foi realizada utilizando-se balança digital (Plena), com capacidade de $150 \mathrm{~kg}$ e sensibilidade de $0,1 \mathrm{~kg}$, com desligamento automático e autocalibragem a cada mensuração.

Para a aferição da estatura, foi utilizada uma fita métrica de aço flexível fixada na parede, tendo sido conferida a sua verticalidade com um fio de prumo. O indivíduo era posicionado nesse local, permanecendo ereto, com pés unidos e calcanhares, nádegas e cabeça em contato com a parede, orientada 
no plano de Frankfurt, registrando-se o valor com precisão de $0,1 \mathrm{~cm}$.

Para a espessura de dobras cutâneas (subescapular, tríceps, bíceps, supra-ilíaca e panturrilha), utilizou-se um compasso de dobras cutâneas, da marca Cescorf, com sensibilidade de 0,1 milímetro.

$\mathrm{O}$ estado nutricional foi avaliado por meio do IMC - massa corporal $(\mathrm{kg}) /$ estatura $\left(\mathrm{m}^{2}\right)$ - e do somatório de espessura de cinco dobras cutâneas ( $55 \mathrm{DC})$. Para verificar a inadequação nutricional, foram definidos pontos de corte segundo recomendações amplamente aceitas na literatura: IMC inadequado $(<18,5$ ou $\geq 25,0$ $\left.\mathrm{kg} / \mathrm{m}^{2}\right)^{19}$ e $\Sigma 5$ DC inadequado $(\leq 30$ ou $\geq 62$ e $\leq 43$ ou $\geq$ $76 \mathrm{~mm}$, para homens e mulheres, respectivamente) ${ }^{20}$.

Para a análise descritiva das variáveis, foram utilizados desvios padrão, médias e proporção. Tabelas de freqüências percentuais foram estabelecidas para verificar a proporção de universitários acima ou abaixo dos pontos de corte adequado para IMC e $\Sigma 5$ DC. Nas comparações entre duas proporções, foi aplicado o teste de significância para diferenças entre proporções. Para verificar as diferenças de freqüência entre as variáveis, utilizou-se o teste qui-quadrado. A associação entre medidas de estado nutricional e insatisfação com a imagem corporal foi analisada por regressão logística. Foram testados dois modelos, um simples e outro múltiplo, controlado por sexo. Em todas as análises foi fixado nível de significância de 5\%. A análise estatística foi realizada utilizando-se o SPSS $^{\circledR}$ versão 10.0 e o MedCalc versão 9.3.3.0.

\section{Resultados}

Os dados da Tabela 1 apresentam a média e o desvio padrão da idade, variáveis antropométricas e percepção atual e ideal da imagem corporal da população estudada.

Tabela 1 - Características físicas, antropométricas e percepção da imagem corporal de universitários segundo sexo (Florianópolis, 2007)

\begin{tabular}{lcccccc}
\hline \multirow{2}{*}{ Variáveis } & \multicolumn{2}{c}{ Masculino } & \multicolumn{2}{c}{ Feminino } & \multicolumn{2}{c}{ Total } \\
\cline { 2 - 7 } & Média & DP & Média & DP & Média & DP \\
\hline Idade (anos) & 22,7 & 3,4 & 23,6 & 6,7 & 23,1 & 5,3 \\
Estatura (cm) & 176,4 & 7,5 & 163,8 & 7,2 & 170,1 & 9,7 \\
Massa corporal (kg) & 70,5 & 11,4 & 58,1 & 10,1 & 64,3 & 12,4 \\
IMC $\left(\mathrm{kg} / \mathrm{m}^{2}\right)$ & 22,6 & 2,8 & 21,6 & 3,1 & 22,1 & 3,0 \\
I5DC & 65,5 & 28,2 & 95,0 & 29,9 & 80,3 & 32,6 \\
Silhueta atual & 3,8 & 1,3 & 3,8 & 1,0 & 3,8 & 1,1 \\
Silhueta ideal & 4,0 & 0,8 & 3,1 & 0,6 & 3,5 & 0,8 \\
\hline
\end{tabular}

$\mathrm{DP}=$ desvio padrão; $\mathrm{IMC}=$ índice de massa corporal; $\Sigma 5 \mathrm{DC}=$ somatório de espessura de cinco dobras cutâneas.

Os resultados demonstraram que a maioria dos indivíduos $(78,8 \%)$ estava insatisfeita com a própria imagem corporal. Além disso, observou-se que 49,2\% dos indivíduos apresentaram desejo de reduzir o tamanho da silhueta, enquanto que $26,6 \%$ desejavam aumentar. A distribuição dos universitários por sexo, segundo satisfação com a imagem corporal, é apresentada na Figura 1.

Os resultados da Figura 1A mostraram que, quando analisados de forma dicotômica, não houve diferença estatística entre homens e mulheres em relação à prevalência de indivíduos insatisfeitos com a imagem corporal. Contudo, quando o grupo de insatisfeitos foi dividido de acordo com o desejo de modificar o tamanho da silhueta, o qui-quadrado indicou maior proporção no sexo feminino $(\mathrm{p}<0,001)$ para o desejo de reduzir as dimensões corporais, enquanto que no sexo masculino existiu um desejo maior para aumentar $(\mathrm{p}<0,001)$ (Figura 1B).

A Figura 2 mostra a proporção de indivíduos classificados segundo o estado nutricional a partir dos pontos de corte para IMC e $55 \mathrm{DC}$. De acordo com os dados da Figura 2A, foi verificado que $83,2 \%$ dos indivíduos foram classificados como eutróficos segundo o IMC, enquanto que no $\Sigma 5$ DC apenas 34\% estavam nessa condição. A diferença nas proporções foi estatisticamente significativa $(p<0,05)$. Quando a análise foi feita de forma não-dicotômica (Figura 2B), verificou-se que o $\Sigma 5 \mathrm{DC}$ indicou maior proporção de sujeitos com sobrepeso $(\mathrm{p}<0,05)$. 


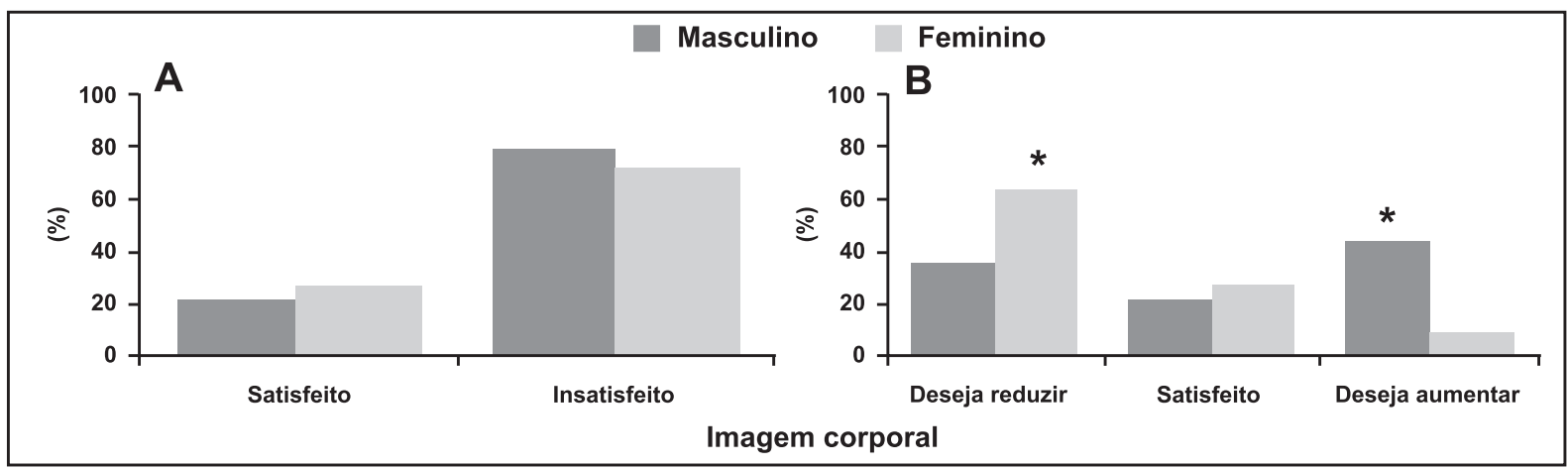

$\mathrm{A}=$ imagem corporal categorizada de forma dicotômica $\left(\chi^{2}=1,362 ; \mathrm{p}=0,243\right) ; \mathrm{B}=\mathrm{o}$ grupo insatisfeito foi transformado em duas categorias: deseja reduzir e deseja aumentar $\left(* \chi^{2}=39,789 ; \mathrm{p}<0,001\right)$.

Figura 1 - Distribuição dos universitários de acordo com a percepção da imagem corporal e sexo (Florianópolis, 2007)

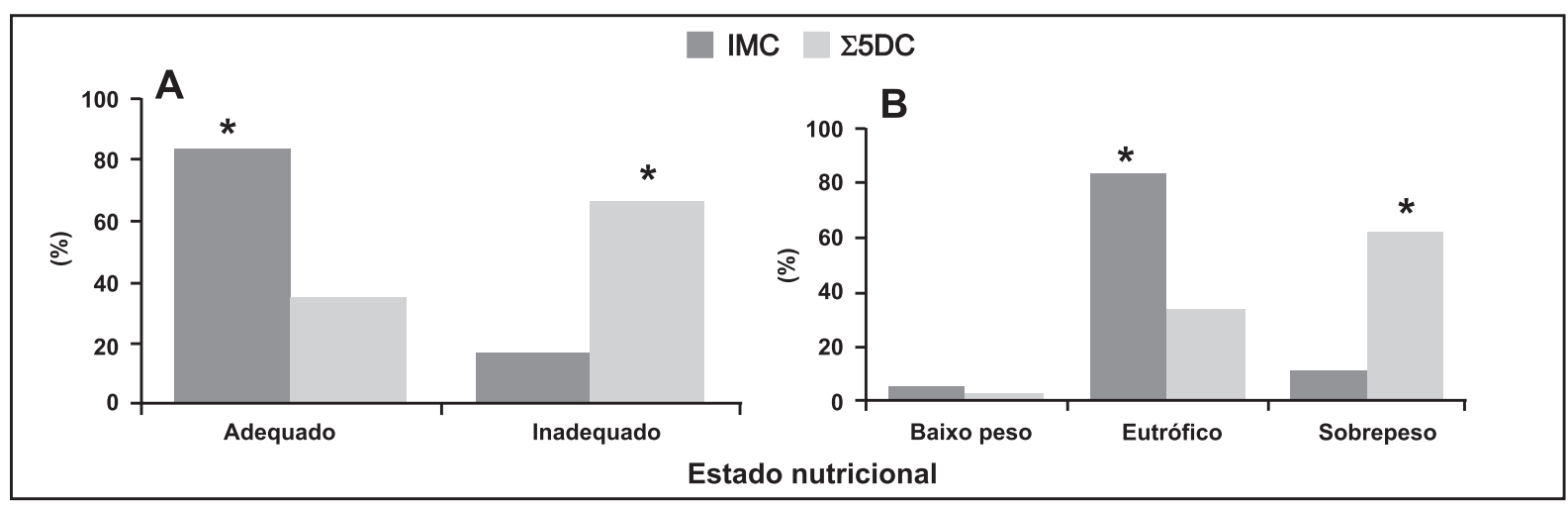

$\mathrm{A}$ = estado nutricional categorizado de forma dicotômica; $\mathrm{B}=\mathrm{o}$ grupo inadequado foi transformado em duas categorias (baixo peso e sobrepeso).

$\Sigma 5 \mathrm{DC}=$ somatório de espessura de cinco dobras cutâneas; IMC = índice de massa corporal.

* $\mathrm{p}<0,05$ para o teste de significância para diferenças entre proporções.

Figura 2 - Distribuição dos universitários de acordo com a classificação do estado nutricional por IMC e $\Sigma 5 \mathrm{DC}$

(Florianópolis, 2007)

A Figura 3 apresenta a proporção dos indivíduos, segundo o sexo, que se situaram abaixo ou acima dos pontos de cortes estabelecidos como adequados para a saúde.

Foi verificada correlação significativa entre IMC e $\Sigma 5 \mathrm{DC}$ para homens $(\mathrm{r}=0,692 ; \mathrm{p}<0,001)$ e mulheres $(\mathrm{r}=0,761 ; \mathrm{p}<0,001)$. O teste de proporção mostrou diferença significativa $(\mathrm{p}<0,05)$ para o $\Sigma 5 \mathrm{DC}$ na distribuição do estado nutricional, com as mulheres apresentando maior prevalência de inadequação nutricional. O mesmo não foi observado para o IMC $(\mathrm{p}>0,05)$ (Figura 3A). Contudo, quando o grupo inadequado foi transformado em duas categorias (baixo peso e sobrepeso), observou-se que um maior número de mulheres foi classificado com baixo peso pelo IMC $(\mathrm{p}<0,05)$, enquanto que pelo $\Sigma 5 \mathrm{DC}$ ocorreu o inverso: maior número de mulheres, que de homens, apresentou sobrepeso $(p<0,05)$ (Figura 3B).

A Tabela 2 mostra a associação entre insatisfação com a imagem corporal e inadequação nutricional de acordo com os dois indicadores antropométricos. A odds ratio (OR) indicou que não houve diferença na freqüência de insatisfação com a imagem corporal entre os sexos (IC95\% 0,40-1,26). A OR bruta indicou 


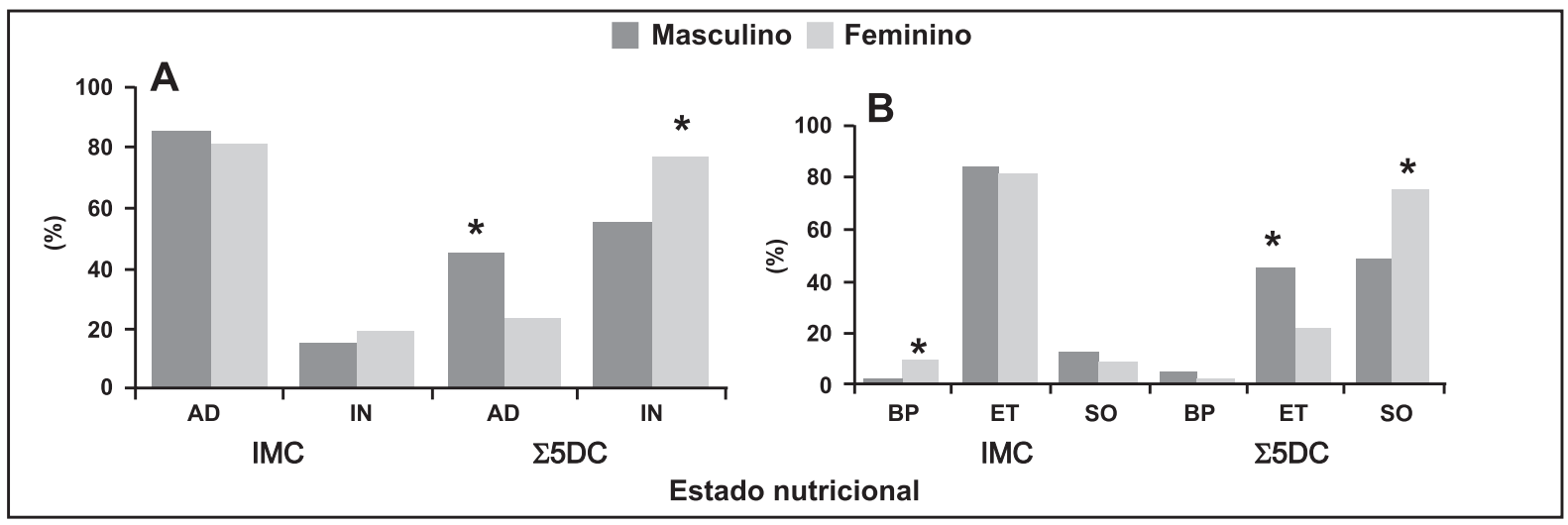

$\mathrm{A}$ = estado nutricional categorizado de forma dicotômica; $\mathrm{B}=\mathrm{o}$ grupo inadequado foi transformado em duas categorias (baixo peso e sobrepeso).

$\mathrm{AD}=$ adequado; $\mathrm{BP}=$ baixo peso $; \mathrm{ET}=$ eutrófico; $\Sigma 5 \mathrm{DC}=$ somatório de espessura de cinco dobras cutâneas; $\mathrm{IMC}=$ índice de massa corporal; IN = inadequado; $\mathrm{SO}=$ sobrepeso.

$* \mathrm{p}<0,05$ para o teste de significância para diferenças entre proporções.

Figura 3 - Distribuição dos universitários de acordo com a classificação do estado nutricional (IMC e $\Sigma 5 \mathrm{DC}$ ) e sexo (Florianópolis, 2007)

que o IMC inadequado $\left(<18,5\right.$ ou $\left.\geq 25,0 \mathrm{~kg} / \mathrm{m}^{2}\right)$ associou-se à insatisfação com a imagem corporal (IC95\% 1,04-7,38), o mesmo ocorrendo com o $\Sigma 5 \mathrm{DC}$ (IC95\% 1,25-4,05). No entanto, no modelo múltiplo ajustado por sexo, apenas o $\Sigma 5 \mathrm{DC}$ inadequado foi preditor significante de insatisfação com a imagem corporal entre os universitários que demonstraram inadequação nutricional, possuindo, segundo esse indicador, duas vezes e meia mais chances de serem insatisfeitos com o próprio corpo (IC95\% 1,36-4,80) do que aqueles que possuíam adiposidade corporal dentro dos níveis adequados à saúde.

Tabela 2 - Odds ratio para insatisfação com a imagem corporal* em universitários segundo indicadores do estado nutricional (Florianópolis, 2007)

\begin{tabular}{lcccc}
\hline Variáveis & OR & IC95\% & $\mathbf{O R}^{\dagger}$ & $\mathbf{I C 9 5 \%}^{\dagger}$ \\
\hline IMC & & & & \\
$\quad$ Adequado & 1,0 & & 1,0 & \\
$\quad$ Inadequado & 2,77 & $1,04-7,38$ & 2,66 & $0,98-7,25$ \\
$\begin{array}{c}\Sigma \text { DDC } \\
\quad \text { Adequado }\end{array}$ & 1,0 & & & \\
$\quad$ Inadequado & 2,25 & $1,25-4,05$ & 2,56 & $1,36-4,80$ \\
\hline
\end{tabular}

IC95\% = intervalo de confiança de 95\%; IMC = índice de massa corporal; $\Sigma 5 \mathrm{DC}=$ somatório de espessura de cinco dobras cutâneas; $\mathrm{OR}=$ odds ratio.

* OR e IC95\% para a variável sexo foram de $0,71(0,40-1,26)$.

${ }^{\dagger}$ Ajustada para sexo. 


\section{Discussão}

De acordo com os achados da literatura, nenhuma pesquisa foi encontrada considerando mais de um indicador antropométrico para verificar a associação entre insatisfação com a imagem corporal e estado nutricional, assim como identificar qual índice antropométrico é o melhor preditor para esse desfecho. Dessa forma, o presente estudo foi o primeiro a tratar da associação entre dois indicadores de estado nutricional e insatisfação com a imagem corporal.

No presente estudo, foram analisados dados de 256 universitários matriculados na disciplina de Educação Física Curricular de uma universidade federal da região de Florianópolis (SC), o que corresponde a $24,8 \%$ da população total.

O método de silhueta corporal utilizado é de fácil e rápida aplicação, simples de ser realizado e tem mostrado boa fidedignidade, sendo bastante utilizado nos últimos anos, inclusive em estudos populacionais ${ }^{4,12,21-23}$ e em universitários ${ }^{11}$. Porém, as principais limitações referentes aos desenhos das silhuetas estão diretamente relacionadas às formas lineares bidimensionais, que podem implicar falhas na representação total do corpo, na distribuição da massa de gordura e em outros aspectos da composição corporal que são importantes na formação da imagem corporal ${ }^{8,24}$. Além disso, é baseado em biótipo que pode não ser o mesmo da população brasileira.

Os resultados mostraram que grande número de universitários está insatisfeito com a própria imagem corporal. Em estudos realizados no Brasil, a prevalência de insatisfação com a imagem corporal foi similar à do presente estudo, sendo descritas prevalências de $82 \%$ em escolares ${ }^{4}$ e de $76 \%$ e $82 \%$ em mulheres e homens fisicamente ativos, respectivamente ${ }^{8}$.

As mulheres, de maneira geral, anseiam pela redução do tamanho da silhueta corporal, enquanto que a predominância nos homens é pelo desejo de ter um corpo mais forte e volumoso. Esses resultados são similares aos de Damasceno et al. ${ }^{8}$, que demonstraram a mesma tendência em estudo realizado com amostra de 186 praticantes de caminhada de uma cidade do estado de Minas Gerais, Brasil.

Essa diferença nas preferências das silhuetas corporais entre homens e mulheres pode ser explicada pelo fato de as mulheres superestimarem a imagem corporal e os homens a subestimarem ${ }^{11,12,22,25-27}$.

Um dado importante encontrado no presente estudo foi a diferença significativa entre os dois indicadores (IMC e $\Sigma 5$ DC) na classificação do estado nutricional. $\mathrm{O}$ teste de Pearson indicou correlação moderada a alta entre os dois indicadores antropométricos em homens e mulheres. Os resultados mostraram ainda que, enquanto o IMC classificou a maioria dos universitários como adequados, o $\Sigma 5 \mathrm{DC}$ indicou que mais da metade destes se encontravam em estado de inadequação nutricional. Essa inconsistência também foi verificada em investigação realizada com adolescentes, que demonstrou que somente $49 \%$ das moças e $57 \%$ dos rapazes foram classificados concomitantemente pelo IMC e somatório de dobras cutâneas $^{15}$.

Embora os resultados do presente estudo tenham mostrado maior prevalência de insatisfação com a imagem corporal nos homens, a análise de regressão indicou que o sexo não foi um preditor significativo de insatisfação com a imagem corporal.

Nos últimos anos, vários estudos transversais têm sido realizados com intuito de verificar a relação entre insatisfação com a imagem corporal e estado nutricional. Porém, as investigações têm utilizado o IMC como a medida padrão de estado nutricional para esse objetivo ${ }^{4,9-12}$, e os resultados não têm se mostrado consistentes.

Nas análises univariadas, observou-se associação significativa entre IMC e insatisfação com a imagem corporal, sendo que os indivíduos com inadequação nutricional possuíam, aproximadamente, três vezes mais chances de desenvolver insatisfação com a própria imagem corporal em relação aos indivíduos adequados. $\mathrm{O}$ mesmo ocorreu com o outro indicador ( $\Sigma 5 \mathrm{DC})$. Entretanto, quando as variáveis (IMC e $\Sigma 5 \mathrm{DC}$ ) foram ajustadas no modelo múltiplo controlado por sexo, a associação entre IMC e o desfecho desapareceu, enquanto a associação do $\Sigma 5 \mathrm{DC}$ se manteve (Tabela $2)$. Esses resultados sugerem que o $\Sigma 5 \mathrm{DC}$ foi o indicador com melhor poder de predição da insatisfação com a imagem corporal em universitários, quando comparado ao IMC.

Os achados referentes ao IMC sugerem que esse indicador nem sempre condiz com a forma como os indivíduos percebem seus corpos. Dessa maneira, existe a possibilidade de um sujeito apresentar um IMC adequado e desejar reduzir ou aumentar o tamanho da silhueta, melhorando a sua satisfação com a imagem corporal. Nesse sentido, parece que o motivo de não ter sido observada associação entre IMC e insatisfação com a imagem corporal foi que grande parte dos indivíduos, independentemente da classificação do IMC (adequado ou inadequado), apresentou insatisfação com o seu tamanho e forma corporais.

Dessa forma, supõem-se duas possíveis explicações para o fato de o $\Sigma 5 \mathrm{DC}$ ter apresentado maior poder de predição de insatisfação com a imagem corporal do que o IMC: 1) como o $55 \mathrm{DC}$ classificou a maioria dos universitários como possuindo excesso de gordura corporal, a insatisfação com a imagem corporal dependeria mais 
da quantidade de tecido adiposo subcutâneo do que da quantidade de massa corporal total; 2) o $\Sigma 5 \mathrm{DC}$, por ser derivado de medidas da gordura corporal subcutânea, é um indicador do estado nutricional e da composição corporal, diferente do IMC, que apenas verifica o estado nutricional.

Vale ressaltar que, ao contrário de algumas pesquisas ${ }^{10,11}$ que sugeriram relação entre IMC e insatisfação com a imagem corporal, os resultados do presente estudo mostraram que o IMC não foi um determinante para o desfecho.

Este trabalho apresenta limitações inerentes aos estudos transversais, ou seja, estima as relações entre as variáveis em apenas um único momento, não permitindo identificar as relações de causa e efeito. É importante destacar também que, no presente estudo, sobrepeso e obesidade foram agrupados juntamente com baixo peso, formando a categoria IMC inadequado. O mesmo critério foi adotado para o $\Sigma 5 \mathrm{DC}$. Dessa forma, a maneira como as variáveis foram dicotomizadas pode não ter sido a melhor forma para evidenciar associação entre estado nutricional e insatisfação com a imagem corporal.

Em suma, a insatisfação com a imagem corporal foi um fenômeno altamente freqüente em universitários de ambos os sexos. Em razão disso, deve ser considerado como um sério problema de saúde pública, pois pode condicionar, em parte, atitudes e condutas comportamentais não-saudáveis, como anorexia, bulimia, dismorfia e aceitação das dietas malorientadas ${ }^{3}$.

De acordo com as análises realizadas, os dados sugerem que, independentemente do sexo, o $\Sigma 5 \mathrm{DC}$ é um forte preditor de insatisfação com a imagem corporal em universitários, enquanto que o IMC parece não ser um determinante significativo desse desfecho. Entretanto, outros fatores comportamentais, principalmente os relacionados aos padrões de estética, devem ser investigados.

Considerando que o presente estudo foi transversal e restrito a universitários, sugerem-se estudos com intervenção nutricional e/ou exercício físico, objetivando determinar o impacto destes na melhoria da composição corporal e, assim, sobre a auto-estima e os níveis de satisfação com a imagem corporal. Outras avaliações comparativas, considerando diferentes grupos sociais e étnicos, são necessárias para maior entendimento quanto à relação entre estado nutricional e imagem corporal.

Finalmente, vale ressaltar que, como forma de oferecer retorno aos participantes da pesquisa, imediatamente após a coleta de dados, foi emitido e entregue, a cada um dos participantes, um laudo com todas as informações e interpretações referentes à composição corporal, com as devidas recomendações.

\section{Referências}

1. Slade PD. Body image in anorexia nervosa. Br J Psychiatry Suppl. 1988;2:20-2.

2. Sepúlveda AR, Botella J, León JA. Body-image disturbance in eating disorders: A meta-analysis. Psychology in Spain. 2002;6:8395.

3. McCabe MP, Ricciardelli LA. Body image dissatisfaction among males across the lifespan: a review of past literature. J Psychosom Res. 2004;56:675-85.

4. Pinheiro AP, Giugliani ER. Body dissatisfaction in Brazilian schoolchildren: prevalence and associated factors. Rev Saude Publica. 2006;40:489-96.

5. Hayashi F, Takimoto H, Yoshita K, Yoshiike N. Perceived body size and desire for thinness of young Japanese women: a populationbased survey. Br J Nutr. 2006;96:1154-62.

6. Hrabosky JI, Grilo CM. Body image and eating disordered behavior in a community sample of Black and Hispanic women. Eat Behav. 2007;8:106-14.

7. Dionne MM, Davis C. Body image variability: the influence of body-composition information and neuroticism on young women's body dissatisfaction. Body Image. 2004;1:335-49.

8. O’Brien K, Venn BJ, Perry T, Green TJ, Aitken W, Bradshaw A, et al. Reasons for wanting to lose weight: different strokes for different folks. Eat Behav. 2007;8:132-35.

9. Damasceno VO, Lima JRP, Vianna JM, Vianna VRA, Novaes JS. Tipo físico ideal e satisfação com a imagem corporal de praticantes de caminhada. Rev Bras Med Esporte. 2005;11:181-86.

10. Conti MA, Frutuoso MFP, Gambardella AMD. Excesso de peso e insatisfação corporal em adolescentes. Rev Nutr. 2005;18:491-97.

11. Kakeshita IS, Almeida SS. Relação entre índice de massa corporal e a percepção da auto-imagem em universitários. Rev Saude Publica. 2006;40:497-504.

12. Branco LM, Hilário MOE, Cintra IP. Percepção e satisfação corporal em adolescentes e a relação com seu estado nutricional. Rev Psiquiatr Clín. 2006;33:292-6.

13. Acuña K, Cruz T. Avaliação do estado nutricional de adultos e idosos e situação nutricional da população brasileira. Arq Bras Endocrinol Metab. 2004;48:345-61.

14. Anjos LA. Índice de massa corporal (massa corporal.estatura ${ }^{-2}$ ) como indicador do estado nutricional de adultos: revisão da literatura. Rev Saude Publica. 1992;26:431-6.

15. Glaner MF. Índice de massa corporal como indicativo da gordura corporal comparado às dobras cutâneas. Rev Bras Med Esporte. 2005; 11:243-6.

16. Stunkard AJ, Sorenson T, Schlusinger F. Use of the Danish adoption register for the study of obesity and thinness. In: Kety SS, Rowland LP, Sidman RL, Matthysse SW, editors. The genetics of neurological and psychiatric disorders. New York: Raven; 1983. p. 115-20.

17. Alvarez BR, Pavan AL. Alturas e comprimentos. In: Petroski EL, editor. Antropometria: técnicas e padronizações. 3 ed. Blumenau: Nova Letra; 2007. p. 31-44.

18. Benedetti TRB, Pinho RA, Ramos VM. Dobras cutâneas. In: Petroski EL, editor. Antropometria: técnicas e padronizações. 3 ed. Blumenau: Nova Letra; 2007. p. 45-56.

19. World Health Organization. Obesity: preventing and managing the global epidemic. Geneva: WHO; 1998.

20. Canadian Society for Exercise Physiology (CSEF). The Canadian physical activity, fitness and lifestyle appraisal: CSEP's guide to health active living. 2nd ed. Ottawa: CSEF; 2003.

21. Sánchez-Villegas A, Madrigal H, Martínez-González MA, Kearney J, Gibney MJ, Irala J, et al. Perception of body image as indicator of weight status in the European Union. J Hum Nutr Diet. 2001;14:93102 .

22. Madrigal H, Sánchez-Villegas A, Martinez-González MA, Kearney $\mathrm{J}$, Gibney MJ, Irala J, et al. Underestimation of body mass index through perceived body image as compared to self-reported body mass index in the European Union. Public Health. 2000;114:46873.

23. Madrigal Fritsch H, de Irala Estévez J, Martínez González MA, 
Kearney J, Gibney M, Martínez Hernández JA. Percepción de la imagen corporal como aproximación cualitativa al estado de nutrición. Salud Pública Méx. 1999;41:479-86.

24. Kay S. The psychological and anthropometric in body image. In: Norton K, Olds T, editors. Anthropometrica. Sydney: University of New South Wales Press; 1996. p. 217-38.

25. Atalah E, Urteaga C, Rebolledo A. Autopercepción del estado nutricional en adultos de Santiago. Rev Med Chile. 2004;132:1383-8.
26. Veggi AB, Lopes CS, Faerstein E, Sichieri R. Índice de massa corporal, percepção do peso corporal e transtornos mentais comuns entre funcionários de uma universidade no Rio de Janeiro. Rev Bras Psiquiatr. 2004;26:242-7.

27. Tanaka S, Itoh Y, Hattori K. Relationship of body composition to body-fatness estimation in Japanese university students. Obes Res. 2002;10:590-6. 